\title{
c-ment Report \\ A Case of High-grade Arteriovenous Malformation Manifesting as Trigeminal Neuralgia Successfully Treated by Embolization in Multimodal Treatment
}

Miyahito Kugai, ${ }^{1}$ Takehiro Suyama, ${ }^{2}$ Masahiko Kitano, ${ }^{1}$ Hiroshi Hasegawa, ${ }^{1}$ Yoshiko Tominaga, ${ }^{1}$

and Shinsuke Tominaga ${ }^{1}$

Objective: The authors report a rare case of symptomatic cerebral arteriovenous malformation (AVM) manifesting as trigeminal neuralgia (TGN) that was successfully treated by a multimodal treatment.

Case Presentation: A 68-year-old man presented with right TGN. The symptom progressed with lancinating pain. Brain MRI showed a right temporal lobe AVM with a maximum diameter of approximately $50 \mathrm{~mm}$. A right ectatic tortuous vertebral artery (VA) compressed the root of the right trigeminal nerve. The TGN completely disappeared after the 4th embolization. After the 6th embolization, excision was performed. No recurrence of TGN was observed in the 6 months after its excision.

Conclusion: The present case suggested that treatment of nidus may improve AVM-induced TGN. Embolization was considered to be an effective modality, especially in multimodal treatment for high-grade AVM.

Keywords > trigeminal neuralgia, vertebral artery, arteriovenous malformation, embolization, multimodal treatment

\section{Introduction}

Trigeminal neuralgia (TGN) is a symptom of neurovascular compression syndrome. Generally, it develops due to compression of the root exit zone (REZ) of the trigeminal nerve near the brainstem by a blood vessel which developed arteriosclerosis with aging, and the main responsible blood vessels include the superior cerebellar artery (SCA), anterior inferior cerebellar artery (AICA), and vertebral artery (VA). Symptomatic treatment with drugs, such as carbamazepine, and stereotactic radiosurgery (SRS) with gamma knife are

${ }^{1}$ Department of Neurosurgery, Tominaga Hospital, Osaka, Osaka, Japan

${ }^{2}$ Department of Neurosurgery, Kansai Medical University Medical Center, Moriguchi, Osaka, Japan

Received: January 13, 2019; Accepted: June 23, 2019

Corresponding author: Miyahito Kugai. Department of Neurosurgery, Tominaga Hospital, 1-4-48 Minatomachi, Naniwa, Osaka, Osaka 556-0017, Japan

Email:md002042@yahoo.co.jp

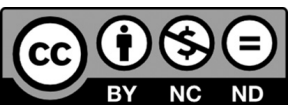

This work is licensed under a Creative Commons Attribution-NonCommercialNoDerivatives International License.

(C)2019 The Japanese Society for Neuroendovascular Therapy performed, and microvascular decompression (MVD) is performed as radical treatment. ${ }^{1)}$ Cerebral arteriovenous malformation (AVM) rarely causes TGN, and many cases treated with direct surgery ${ }^{2-7)}$ and SRS $^{8,9}$ have been reported, but cases treated with embolization ${ }^{4,9-13)}$ have also been occasionally reported. We encountered a rare case of high-grade AVM which manifested as TGN. We performed multimodal treatment and the symptoms were resolved.

\section{Case Presentation}

A 68-year-old male. He developed AVM-induced right TGN about 15 years before. Lancinating pain was induced by food ingestion, washing face, and speech, therefore drug therapy centering on carbamazepine was performed, but the effect was insufficient. The symptoms progressed and a gamma knife was applied to the trigeminal nerve root 2 years earlier, but it was ineffective, and the patient was referred to our hospital. No abnormal neurological finding was noted on the first examination. About 50-mm AVM was detected in the right medial temporal lobe on MRI, MRA, 3DCTA, and cerebral angiography (Fig. 1A and 1B), causing steal phenomenon-induced cerebral edema. The feeders were the middle cerebral artery (MCA), posterior cerebral artery 

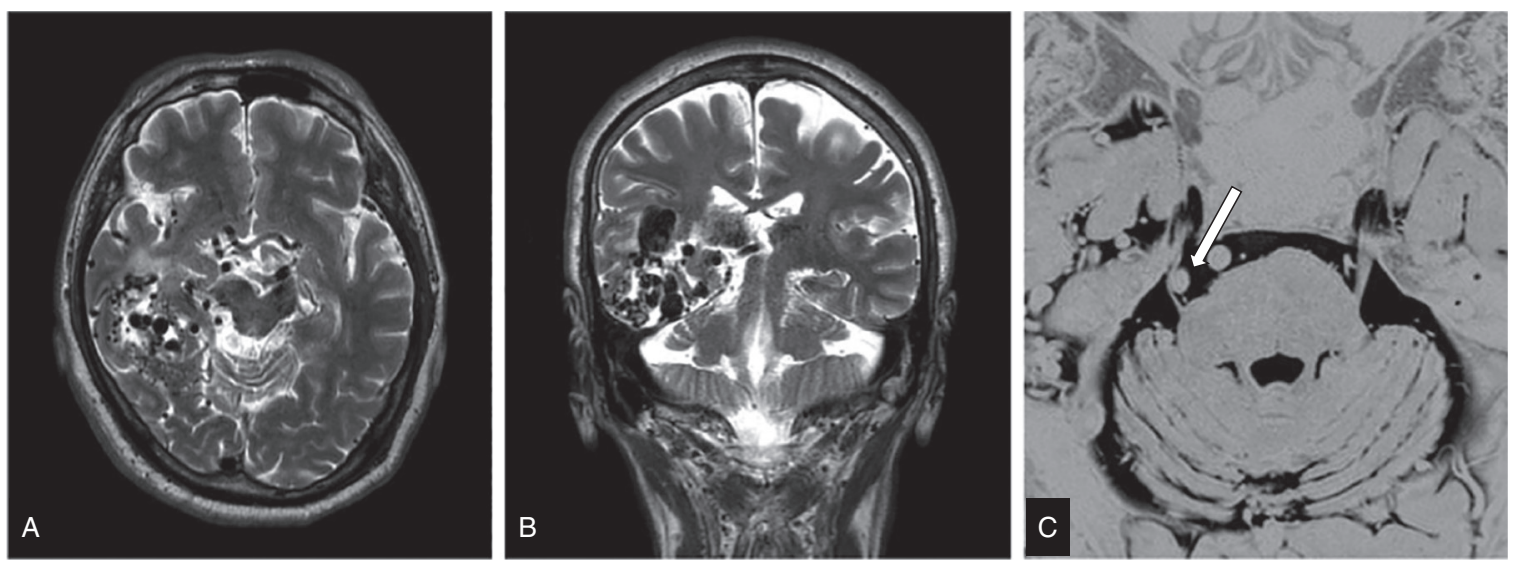

Fig. 1 (A) Axial view on preoperative T2-weighted MRI. (B) Coronal view on preoperative T2-weighted MRI. AVM with a maximum diameter of about $50 \mathrm{~mm}$ was present in the right temporal lobe and it was Grade IV on the SM grading scale. (C) The right VA strongly compressed the right trigeminal nerve from the medial side on preoperative MRI CISS. The trigeminal nerve largely deviated. AVM: arteriovenous malformation; CISS: constructive interference in steady state; SM: Spetzler-Martin; VA: vertebral artery
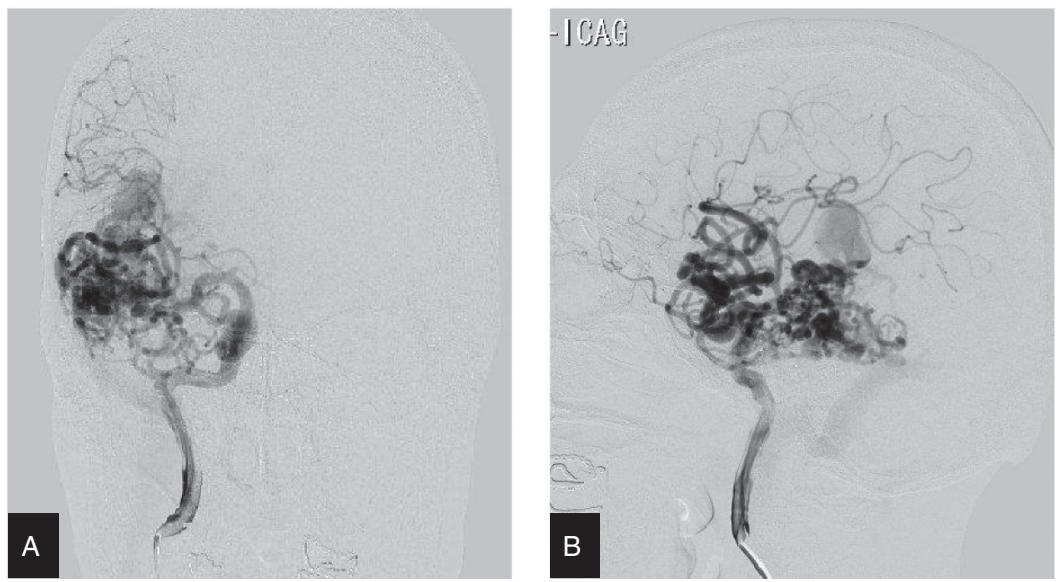

Fig. 2 (A) Frontal view on preoperative angiography of the right internal carotid artery. (B) Lateral view on preoperative angiography of the right internal carotid artery. Many feeders from the right MCA and anterior choroidal artery were present in the right temporal lobe. ICAG: internal carotid angiography; MCA: middle cerebral artery

(PCA), and anterior choroidal artery, and the drainers were the great cerebral vein of Galen, basal vein of Rosenthal, and vein of Labbe, and the condition was Spetzler-Martin (SM) grading scale Grade IV (Figs. 2 and 3). No intranidal aneurysm was noted, but about 2-mm feeder aneurysm was concomitantly present at the origin of the anterior choroidal artery. The right trigeminal nerve largely deviated due to strong compression from the medial side by the dilated tortuous right VA on preoperative head MRI constructive interference in steady state (CISS) (Fig. 1C). Based on these findings, compression of the trigeminal nerve by VA was considered the cause of TGN. Since the symptoms were not remitted by medication or SRS, surgical treatment of TGN was investigated. MVD of the VA by craniotomy was considered, but we wanted to eliminate progression of the steal phenomenon and risk of AVM hemorrhage in consideration of the presence of the steal phenomenon, deep localization, associated aneurysm, and deep venous drainage of the main drainer. ${ }^{14,15)}$ Since TGN was also present, a treatment strategy was comprehensively investigated corresponding to the symptoms. It has been reported that treatment of nidus reduced blood flow and the size of the feeder, which resolved pulsatile compression of the trigeminal nerve and remitted TGN. ${ }^{4,9-13)}$ Since the present case had high-grade AVM, nidus treatment was included in multimodal treatment aiming at elimination of the hemorrhagic risk and treatment of TGN. It was planned to perform excision after multistage embolization and add MVD as the 

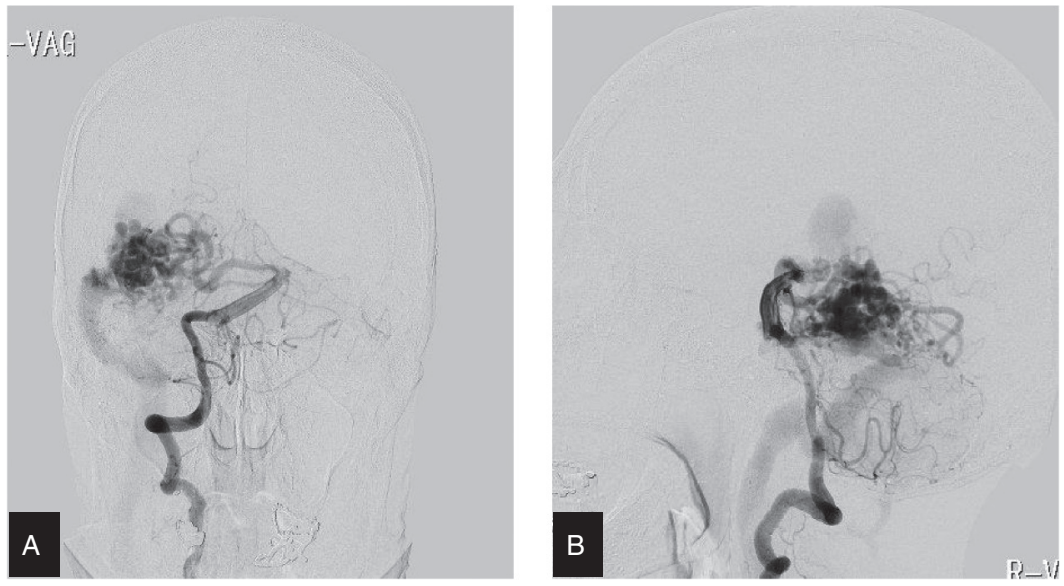

Fig. 3 (A) Frontal view on preoperative angiography of the right VA. (B) Lateral view on preoperative angiography of the right VA. Many feeders from the right PCA were present in the right temporal lobe, and the right VA was dilated and tortuous. PCA: posterior cerebral artery; VA: vertebral artery; VAG: vertebral arteriography
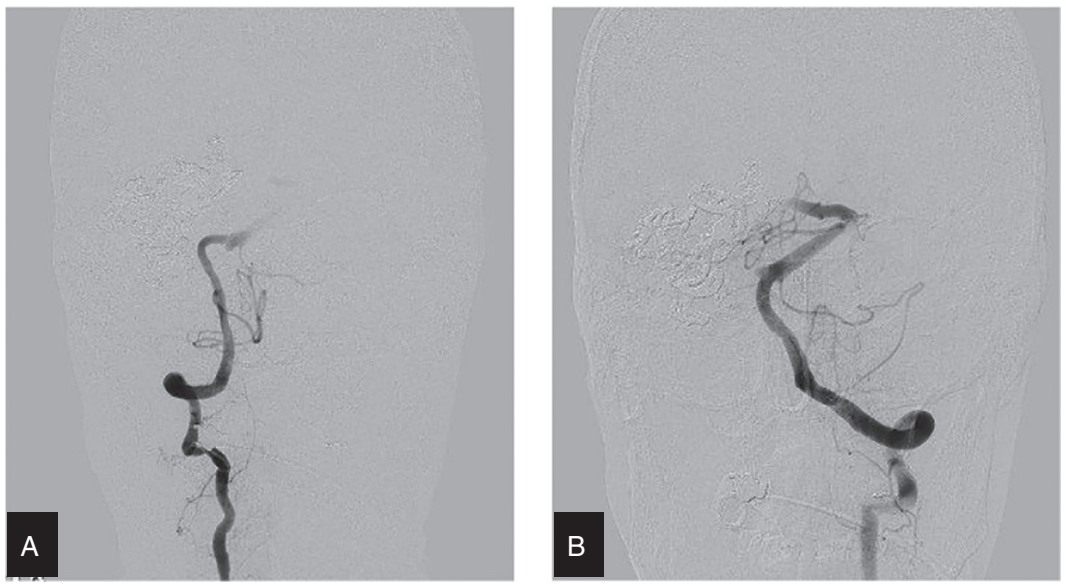

Fig. 4 (A) Frontal view on angiography of the right VA. (B) Frontal view on angiography of the left VA. After the 4th embolization. AVM was no longer visualized through the posterior circulation. AVM: arteriovenous malformation; VA: vertebral artery

second step in case TGN did not improve. Excision was performed after 6 stages of embolization applied at 1- to 3-month intervals.

Under general anesthesia, embolization with n-butyl2-cyanoacrylate (NBCA, Histoacryl; B.Braun, Melsungen, Germany) and Onyx Liquid Embolic System (Onyx; ev3 Neurovascular, Covidien, Plymouth, MN, USA) was performed. The feeders from the MCA and those from the PCA were embolized with NBCA and Onyx in the 1st and 2nd-4th stages, respectively. AVM could be mostly embolized with Onyx in the 4th stage and it was no longer visualized through the posterior circulation (Fig. 4). TGN was not improved by the 3rd embolization, but it improved after the 4th embolization. TGN completely resolved after about 1.5 months and carbamazepine administration was discontinued. Subsequently, the feeders from the MCA and anterior choroidal artery were embolized with NBCA in the 5th and 6th embolization, respectively, and AVM was almost completely occluded on angiography. The interval between the 4th and 6th embolization was about 5 months, but no symptom recurred throughout this period.

AVM could be completely excised by craniotomy 3 days after the final embolization (Fig. 5A). MVD was not added because disappearance of TGN continued after surgery. On postoperative head MRI CISS, compression of the right trigeminal nerve by the right VA continued (Fig. 5B). At 3 weeks after surgery, the patient was discharged at modified Rankin Scale (mRS) 1 although mild cerebellar ataxia remained, it improved on subsequent follow-up. As of 6 months after excision, no TGN has recurred. The feeder 

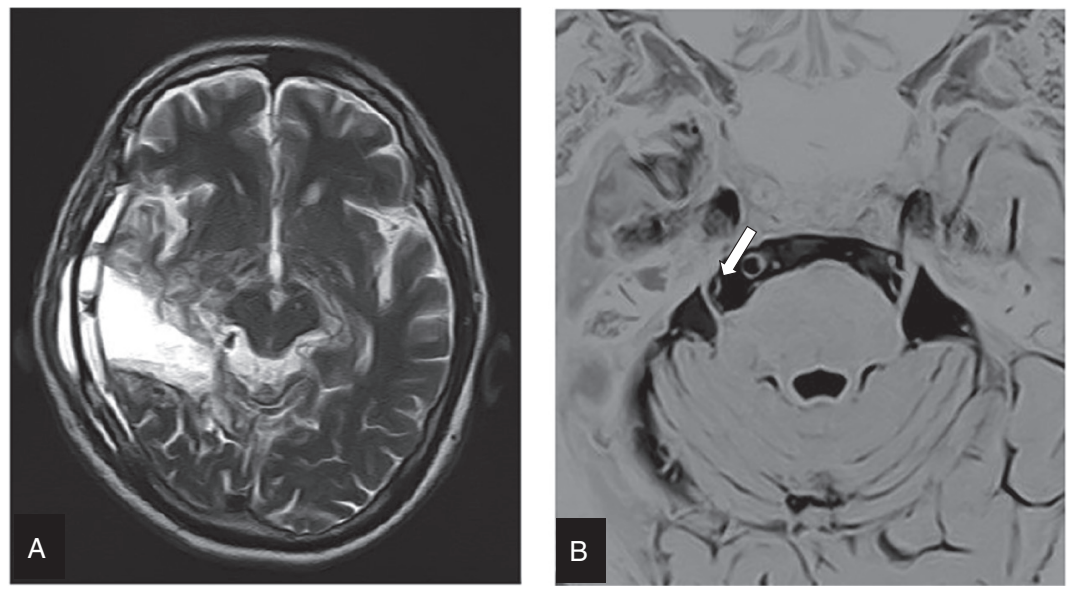

Fig. 5 (A) AVM was totally excised on postoperative T2-weighted MRI. (B) MRI CISS after excision. No marked change was noted in the right VA strongly compressing the trigeminal nerve from the medial side, but TGN resolved. AVM: arteriovenous malformation; CISS: constructive interference in steady state; TGN: trigeminal neuralgia; VA: vertebral artery

aneurysm of the anterior choroidal artery has not changed and it is being followed.

\section{Discussion}

AVM-induced TGN accounts for $0.22-1.78 \%$ of primary TGN cases and the causative AVM is located in the posterior cranial fossa in $97.5 \% .{ }^{13)}$ The most frequent responsible blood vessel is the SCA serving as a feeder, ${ }^{12}$ and compression by nidus and a dilated drainer has also been reported. ${ }^{7,8,12,13)}$ No treatment strategy has been established for AVM manifesting as TGN and it has to be investigated in each case. Regarding direct surgery, (1) treatment with MVD alone without treating AVM, ${ }^{5,7)}$ (2) only AVM excision without MVD, ${ }^{2)}(3)$ simultaneous excision and MVD, ${ }^{2)}$ (4) SRS following MVD, ${ }^{6}$ (5) MVD following SRS, ${ }^{3)}$ and (6) excision following embolization ${ }^{4}$ have been reported. Edwards et al. $\left.{ }^{2}\right)$ recommended AVM excision to not only remit symptoms but also eliminate hemorrhagic risk, and our treatment strategy follows this. Wanke et al. ${ }^{4}$ also added excision to prevent recurrence of TGN even though symptoms improved after embolization. In contrast, García-Pastor et al. ${ }^{5)}$ reported that AVM excision is not always necessary, MVD alone is the optimum. In any case, symptoms disappeared or tended to remit immediately after treatment, suggesting the importance of flexibly investigating treatment for TGN in consideration of hemorrhagic and surgical risks, not sticking to excision. In low-grade AVM, TGN may be simply resolved by excision alone. In high-grade AVM, nidus treatment may not be safely completed using only one modality and it is desirable to perform multimodal treatment. When symptoms are not improved by multimodal treatment, application of MVD should be investigated. Other than direct surgery, it has been occasionally reported that (7) SRS alone was effective, ${ }^{8)}(8)$ embolization alone was effective, ${ }^{10-13)}$ and (9) SRS was performed after embolization. ${ }^{9)}$ When the risks of excision and MVD are expected to be high, noninvasive modalities, such as SRS and embolization, may be investigated. TGN may also be resolved in cases which may be cured by embolization alone. For high-grade AVM difficult to treat, multimodal treatment combining SRS and embolization may be an effective option. However, for high-grade AVM at grades III and IV on the SM grading scale, the morbidity and mortality accompanying surgical treatment has been reported to be about $15-40 \%{ }^{16)}$ Since incomplete treatment increases the hemorrhage rate compared with that in natural history, a treatment strategy has to be prepared in consideration of a state finally requiring excision. ${ }^{17,18)}$ Therefore, although symptoms may be improved by partial embolization reducing blood flow, easygoing treatment with embolization alone must be strictly refrained from. The hemorrhage rate in high-grade AVM is controversial ${ }^{19)}$ and it depends on the condition, so that investigation is required in each case.

Embolization does not target occlusion of the responsible blood vessel directly compressing the trigeminal nerve, but it is applied targeting nidus. It results in reduction of the size of not only nidus but also the feeder due to reduction of blood flow, which resolves pulsatile compression of the trigeminal 
nerve and remits TGN relatively early, suggesting the efficacy of embolization. ${ }^{9-13)}$ Yuan et al. ${ }^{13)}$ reported that TGN completely resolved 1 week after treatment. It has been reported that even when the responsible blood vessel is a dilated drainer, a similar symptom-improving effect of embolization was acquired. ${ }^{12)}$ On the other hand, it has been suggested that a long-term effect may not be expected and repeating embolization several times was required for treatment of TGN which recurred with re-canalization of AVM. ${ }^{11)}$ In another reported case, the addition of treatment other than excision to embolization improved symptoms. Mori et al. $\left.{ }^{9}\right)$ reported a case in which symptoms remitted after embolization, subsequently SRS was applied to nidus, and the symptoms disappeared, concluding that this was due to remission of dilatation of the SCA, the responsible blood vessel, with regression of nidus. In the present case, visualization through the posterior circulation disappeared after the 4th embolization and then TGN completely resolved after 1.5 months. The 4 th embolization may have reduced blood flow in the posterior circulation and remitted pulsatile compression of the responsible blood vessel, right VA. It was suggested that high-grade AVM-induced TGN may also be resolved by multistage embolization, confirming the usefulness of embolization in multimodal treatment. On head MRI CISS after excision (Fig. 5B), compression of the right trigeminal nerve by the right VA remained on imaging, but pulsation on the trigeminal nerve may have decreased as VA blood flow decreased, resolving TGN. It was also suggested that even though AVM is not present in the posterior cranial fossa and it is located at a site distant from the trigeminal nerve, as noted in this case, embolization of AVM may resolve TGN by reducing blood flow and pulsation.9) The objective of embolization includes making excision easier by decompression of nidus, prevention of the normal perfusion pressure breakthrough (NPPB) phenomenon, and processing of a deep feeder difficult to secure in the surgical field. The surgical field was almost bloodless in excision in this case. The usefulness of embolization in multimodal treatment of high-grade AVM may be very high.

It was suggested that in addition to MVD aiming at direct improvement of TGN, excision of AVM, embolization, and SRS or a combination of these may reduce blood flow in AVM and remit or resolve TGN. Embolization to treat AVM-induced TGN has recently been increasingly reported and its role has been expanding. However, when treatment with embolization is planned, nidus should also be treated as much as possible not limiting the aim to improvement of TGN. When nidus remained, it is necessary to carefully investigate additional treatment with excision, embolization, and SRS. When complete removal of nidus in high-grade AVM is considered difficult, treatment with MVD alone from the beginning should be considered as an option.

\section{Conclusion}

For AVM-induced TGN, it was suggested that treatment of nidus decreases blood flow and reduces the size of the feeder, which remits pulsatile compression on the trigeminal nerve and improves TGN. Embolization may be a useful modality especially for multimodal treatment of high-grade AVM. However, it is important to flexibly investigate treatment for TGN in consideration of the hemorrhagic and surgical risks.

\section{Disclosure Statement}

None of the first and coauthors have conflicts of interest.

\section{References}

1) Matsushima $T$, Mineta $\mathrm{T}$ : [Microvascular compression syndrome: review and update of microvascular decompression surgery]. No Shinkei Geka 2008; 36: 303-313. (in Japanese)

2) Edwards RJ, Clarke Y, Renowden SA, et al: Trigeminal neuralgia caused by microarteriovenous malformations of the trigeminal nerve root entry zone: symptomatic relief following complete excision of the lesion with nerve root preservation. J Neurosurg 2002; 97: 874-880.

3) Sato K, Jokura H, Shirane R, et al: Trigeminal neuralgia associated with contralateral cerebellar arteriovenous malformation. Case illustration. J Neurosurg 2003; 98: 1318.

4) Wanke I, Dietrich U, Oppel F, et al: Endovascular treatment of trigeminal neuralgia caused by arteriovenous malformation: is surgery really necessary? Zentralbl Neurochir 2005; 66: 213-216.

5) García-Pastor C, López-González F, Revuelta R, et al: Trigeminal neuralgia secondary to arteriovenous malformations of the posterior fossa. Surg Neurol 2006; 66: 207-211; discussion 211.

6) Sumioka S, Kondo A, Tanabe H, et al: Intrinsic arteriovenous malformation embedded in the trigeminal nerve of a patient with trigeminal neuralgia. Neurol Med Chir (Tokyo) 2011; 51: 639-641.

7) Inoue $T$, Shima A, Hirai $H$, et al: Trigeminal neuralgia due to red vein draining a supratentorial arteriovenous malformation: case report. J Neurol Surg Rep 2016; 77: e109-e112. 
8) Anderson WS, Wang PP, Rigamonti D: Case of microarteriovenous malformation-induced trigeminal neuralgia treated with radiosurgery. J Headache Pain 2006; 7: 217-221.

9) Mori Y, Kobayashi T, Miyachi S, et al: Trigeminal neuralgia caused by nerve compression by dilated superior cerebellar artery associated with cerebellar arteriovenous malformation: case report. Neurol Med Chir (Tokyo) 2014; 54: 236-241.

10) Lesley WS: Resolution of trigeminal neuralgia following cerebellar AVM embolization with Onyx. Cephalalgia 2009; 29: 980-985.

11) Simon SD, Yao TL, Rosenbaum BP, et al: Resolution of trigeminal neuralgia after palliative embolization of a cerebellopontine angle arteriovenous malformation. Cent Eur Neurosurg 2009; 70: 161-163.

12) Ge H, Lv X, Jin H, et al: Role of endovascular embolization for trigeminal neuralgia related to cerebral vascular malformation. Interv Neuroradiol 2016; 22: 600-605.

13) Yuan Y, Zhang Y, Luo QI, et al: Trigeminal neuralgia caused by brain arteriovenous malformations: a case report and literature review. Exp Ther Med 2016; 12: $69-80$.
14) Brown RD, Wiebers DO, Forbes GS: Unruptured intracranial aneurysms and arteriovenous malformations: frequency of intracranial hemorrhage and relationship of lesions. J Neurosurg 1990; 73: 859-863.

15) Gross BA, Du R: Natural history of cerebral arteriovenous malformations: a meta-analysis. J Neurosurg 2013; 118: 437-443.

16) Spetzler RF, Zabramski JM: Grading and staged resection of cerebral arteriovenous malformations. Clin Neurosurg 1990; 36: 318-337.

17) Miyamoto S, Hashimoto N, Nagata I, et al: Posttreatment sequelae of palliatively treated cerebral arteriovenous malformations. Neurosurgery 2000; 46: 589-594; discussion 594-595.

18) Han PP, Ponce FA, Spetzler RF: Intention-to-treat analysis of Spetzler-Martin grades IV and V arteriovenous malformations: natural history and treatment paradigm. J Neurosurg 2003; 98: 3-7.

19) Jayaraman MV, Marcellus ML, Do HM, et al: Hemorrhage rate in patients with Spetzler-Martin grades IV and V arteriovenous malformations: is treatment justified? Stroke 2007; 38: $325-329$. 